\title{
Tin clustering and precipitation in the oxide during autoclave corrosion of Zircaloy-2
}

\author{
G. Sundell, M. Thuvander, H.-O. Andrén \\ E-mail: gustav.sundell@chalmers.se \\ Phone: +46-(0)31-772 3291 \\ Dept. of Applied Physics \\ Chalmers University of Technology \\ 41296 Göteborg, Sweden
}

\begin{abstract}
Atom probe tomography has been used to study the evolution of tin distribution during the corrosion process in Zircaloy-2. From being completely soluble in the Zr metal matrix, some clustering is evident already in the newly formed oxide close to the metal-oxide interface. Analysis of thicker oxides a few hundred nanometers away from the interface reveals fully developed precipitates of metallic Sn particles of up to $20 \mathrm{~nm}$ in size. Although the precipitates contain significant amounts of $\mathrm{Zr}$, it is concluded that they are in the process of being depleted in $\mathrm{Zr}$, which is limited only by the slow diffusion in the oxide scale. The findings are interpreted as being a result of the nobility of the Sn yielding a strong driving force to remain in a metallic state after incorporation in the barrier oxide layer. As Sn occupies substitutional sites in the $\mathrm{ZrO}_{2}$ lattice it is oxidized to a 4+ state when incorporated into the oxide, and in order to remain metallic it must nucleate into precipitates within the inner part of the oxide scale before being re-oxidized to $2+$ and eventually to $4+$ when the oxygen activity is sufficiently high in the outer parts of the oxide.
\end{abstract}

Keywords: Zircaloy-2, corrosion, precipitation, atom probe tomography, Sn, zirconium oxide.

\section{Introduction}

Owing to their low thermal neutron capture cross-section, zirconium alloys are used extensively in the nuclear industry as fuel cladding and for structural components in the reactor core. One of the limiting factors for the lifetime of the nuclear fuel assemblies in light water reactors (LWRs) is oxidation of the cladding. Pure zirconium has poor corrosion resistance, but small additions of alloying elements significantly improve the performance as a passive film of $\mathrm{ZrO}_{2}$ forms upon exposure to reactor coolant. A good empirical understanding of the effect of each alloying element has been established over the years, and 
developments within each alloy family now involve only very subtle tinkering with their chemistry.

Waterside corrosion of Zr alloys typically conforms to a cyclic pattern. The rapid build-up of a protective film of approximately 1-3 $\mu \mathrm{m}$ thickness slows down the growth rate, which is believed to be limited by slow oxygen diffusion through the oxide scale [1]. After some time the protective film breaks down, leading to acceleration in oxide growth and the formation of a new protective layer. This process repeats itself until eventually a breakaway oxidation stage is reached where the oxide is no longer protective.

Tin is added to most Zr-alloys used in LWRs as it improves the mechanical behavior of the cladding due to solid solution strengthening. The amounts that are typically added to commercial alloys (up to $1.7 \%$ by mass) are soluble substitutionally in the $\alpha$-Zr matrix, thus not forming any precipitate phases. The impact of $\mathrm{Sn}$ on the corrosion resistance of LWR cladding has been studied intensively recently, and it has been found to have an adverse effect on the oxidation properties. The current trend in alloy development is therefore to reduce the Sn content of the cladding, which has lead to a number of new low Sn, or Sn-free, cladding materials such as Optimized ZIRLO ${ }^{\mathrm{TM}}$ or $\mathrm{M}^{\mathrm{TM}}$, respectively. Garde et al. found that decreasing the Sn content in Zircaloy-4 to the lower limit of the ASTM specification reduces the corrosion rate significantly, which was attributed to slower $\mathrm{O}^{2-}$ diffusion through the oxide scale in the absence of Sn [2]. Wei and co-workers found that $\mathrm{Sn}$ stabilizes the tetragonal $\mathrm{ZrO}_{2}$ phase, which is regarded to have a detrimental effect as the stress relaxation-induced phase transformation to monoclinic may lead to cracking of the oxide and premature transition [3]. This hypothesis was developed further by Frankel et al. who used synchrotron radiation to study the evolution of tetragonal phase fraction with respect to transition [4].

Despite the efforts to elucidate the effects of $\mathrm{Sn}$ on the oxidation behavior, the fate of the alloying element after incorporation into the oxide has not been fully established. Some studies report segregation of Sn to crystallite boundaries in the oxide [5]. Pêcheur et al. investigated the oxidation state of $\mathrm{Sn}$ using Mössbauer spectroscopy, and could identify $\mathrm{Sn}^{2+}$ and $\mathrm{Sn}^{4+}$ in the oxide film, but suggested that some metallic $\beta$-Sn may also be present particularly in pretransition oxide scales [6]. Anada et al. used the same technique but could not find evidence of metallic $S n$ in the oxide [7]. More recently atom probe tomography (APT) data from Dong and co-workers shows evidence of grain boundary segregation and minor rejection of $\mathrm{Sn}$ by the advancing oxide front [8].

With a growing awareness of the effects of Sn addition to Zr-alloys it is crucial to gain a comprehensive understanding of its behavior during the corrosion process. Atomic scale resolution combined with unique sensitivity to all elements makes APT an ideally suited technique to thoroughly investigate the evolution of the $\mathrm{Sn}$ distribution in the $\mathrm{ZrO}_{2}$ scale during the corrosion process. 


\section{Material and experimental details}

The materials that are analyzed are two commercial Zircaloy- 2 cladding tube materials that are heat treated according to the Westinghouse designated LK2 and LK3 scheme, respectively. These are described by the normalized heat treatment parameter $A$, defined as [9]

$$
A=\sum t_{i} \exp \left(-Q / R T_{i}\right)
$$

where $i$ corresponds to the $i$ th heat treatment after $\beta$-quenching at the temperature $T_{i}(\mathrm{~K})$ during the time $t_{i}(\mathrm{~h}), R$ is the gas constant and $Q$ the activation energy ( $63 \mathrm{kcal} / \mathrm{mol}$ or $264 \mathrm{~kJ} / \mathrm{mol})$. Log $A$ for the LK2 is -16.0 and for LK3 -13.9, i.e. LK3 represents a longer equivalent heat treatment time. There is a slight difference in chemistry between the two materials as can be seen in table 1.

Table 1: Nominal compositions of the analyzed materials, by weight.

\begin{tabular}{llllllll}
\hline Material & Sn (\%) & Fe (\%) & Cr (\%) & Ni (\%) & Si (ppm) & O (ppm) & N (ppm) \\
\hline LK2 & 1.46 & 0.12 & 0.10 & 0.05 & 80 & 1240 & 40 \\
LK3 & 1.32 & 0.17 & 0.10 & 0.05 & 70 & 1300 & 50 \\
\hline
\end{tabular}

The mean precipitate size in the LK2 material (including $\operatorname{Zr}(\mathrm{Cr}, \mathrm{Fe})_{2}$ and the slightly larger $\mathrm{Zr}_{2}(\mathrm{Ni}, \mathrm{Fe})$ particles) is $20 \mathrm{~nm}$, whereas it is $76 \mathrm{~nm}$ in the more heavily heat treated LK3 material. The materials were oxidized in steam, using a static autoclave at a temperature of $400^{\circ} \mathrm{C}$, with the exception of the $9 \mu$ m oxide samples that were oxidized at $415^{\circ} \mathrm{C}$. In all cases the pressure was $10.3 \mathrm{MPa}$. Three stages of the corrosion process were investigated in this study; after 3 days of autoclave exposure (oxide thickness approximately $1 \mu \mathrm{m}$ ), 6 and 15 days of autoclave exposure for LK3 and LK2 respectively (oxide thickness around $2 \mu \mathrm{m}$ ) and 30 and 150 days of autoclave exposure for LK3 and LK2 respectively (oxide thickness about $9 \mu \mathrm{m}$ ). Both LK2 and LK3 materials have been used extensively in nuclear power plants over the past decades and comprehensive data of their in-reactor corrosion behavior exists. LK3 exhibits a slightly better corrosion resistance, which has been attributed to the heat treatment scheme leading to larger SPPs than in LK2, leading to a more dense oxide with fewer cracks [10].

Table 2: Autoclave exposure conditions for the studied materials.

\begin{tabular}{llll}
\hline Material & Exposure (days) & Temperature $\left({ }^{\circ} \mathrm{C}\right)$ & Oxide thickness $(\mu \mathrm{m})$ \\
\hline LK2 $1 \mu \mathrm{m}$ & 3 & 400 & 1.3 \\
LK2 $2 \mu \mathrm{m}$ & 6 & 400 & 2.3 \\
LK2 $9 \mu \mathrm{m}$ & 30 & 415 & 8.8 \\
LK3 $1 \mu \mathrm{m}$ & 3 & 400 & 1.0 \\
LK3 $2 \mu \mathrm{m}$ & 15 & 400 & 1.8 \\
LK3 $9 \mu \mathrm{m}$ & 150 & 415 & 9.0 \\
\hline
\end{tabular}


Preparation of needle-shaped specimens for APT analysis was performed using a FEI Versa 3D DualBeam focused ion beam workstation, equipped with a scanning electron microscope (FIB-SEM). The method of APT tip fabrication was the conventional in situ lift-out technique, with subsequent tip sharpening. The details of this method are described elsewhere [11,12]. The tip milling with the $\mathrm{Ga}^{+}$ion beam was carried out at $30 \mathrm{kV}$ using gradually decreasing currents ranging from $240 \mathrm{pA}$ to $6.9 \mathrm{pA}$, which was subsequently followed by a polishing step at $5 \mathrm{kV}$ using a $22 \mathrm{pA}$ current.

The APT analyses were carried out using a laser-assisted local electrode atom probe of model Imago LEAP 3000X HR. Field evaporation of the sample is initiated by laser pulsing with green light (wavelength $532 \mathrm{~nm}$ ) at a frequency of $200 \mathrm{kHz}$. Laser pulse energies were typically $0.25 \mathrm{~nJ}$ when analyzing the metal, and $0.5 \mathrm{~nJ}$ when analyzing the oxide to compensate for the lower absorption. The acquired data was evaluated and analyzed using the CAMECA IVAS 3.6.6 software. The default k-factor of 3.3 was used in the reconstructions, with an evaporation field of $28 \mathrm{~V} / \mathrm{nm}$.

The outer parts of the $\mathrm{ZrO}_{2}$ scale are difficult to analyze with APT for a variety of reasons. Firstly, the oxide scale has poor electric conductivity, poor thermal conductivity, low absorbance of green light and, most importantly, is full of micrometer scale lateral cracks. APT cannot readily be used to analyze volumes containing cracks, and needles will typically fracture in the crack vicinity. This means that the probed volumes are typically located no longer than approximately $500 \mathrm{~nm}$ from the metal-oxide interface.

Zirconium alloys typically form a sub-oxide of approximate composition ZrO when subjected to autoclave corrosion [13]. In the analyzed materials the suboxide thickness varies significantly. For the samples with shorter exposure times (total oxide thickness 1-2 $\mu \mathrm{m}$ ), the sub-oxide thickness ranges between 0 and $50 \mathrm{~nm}$ [10]. In the $9 \mu \mathrm{m}$ oxide thickness samples, the sub-oxide can reach a thickness of several hundred nanometers and have a very irregular morphology. Unless otherwise stated, this paper will be concerned with Sn distributions in the $\mathrm{ZrO}_{2}$ phase where the $\mathrm{Sn}$ distribution is markedly different to what is found in metal and sub-oxide.

In the mass spectra from the oxide, the $\mathrm{Sn}^{2+}$ peaks between 56 and $62 \mathrm{Da}$ do contain some background from the thermal tails of the more abundant $\mathrm{ZrO}^{2+}$ peaks that precede them. This can make the matrix concentration appear erroneously high in the reconstructions. Therefore, clustering can in reality be even more pronounced than what is visible in figures presented in this article. No clear signs of artifacts induced by the laser pulsing during APT analysis of the oxide were observed. The pole retention of $\mathrm{Sn}$ that is a characteristic of the metal was absent in the analyses of the oxide. 


\section{Results}

\section{Sn in the metal}

Atom maps from APT of the Sn distribution in the Zr metal matrix reveal that, as is expected, $\mathrm{Sn}$ is distributed homogeneously in the matrix. However, having a slightly higher evaporation field than the host Zr lattice [14], Sn may be retained somewhat at the APT specimen surface, diffuse along the surface and eventually field evaporate at crystallographic poles. This leads to an apparent elevated Sn concentration at the pole, which is not a real effect but an artifact generated by the field evaporation process. However, this phenomenon can be utilized to ascertain that an analysis has traversed a grain boundary, where the position of the pole changes as a new grain begins to field evaporate (see Figure 1).

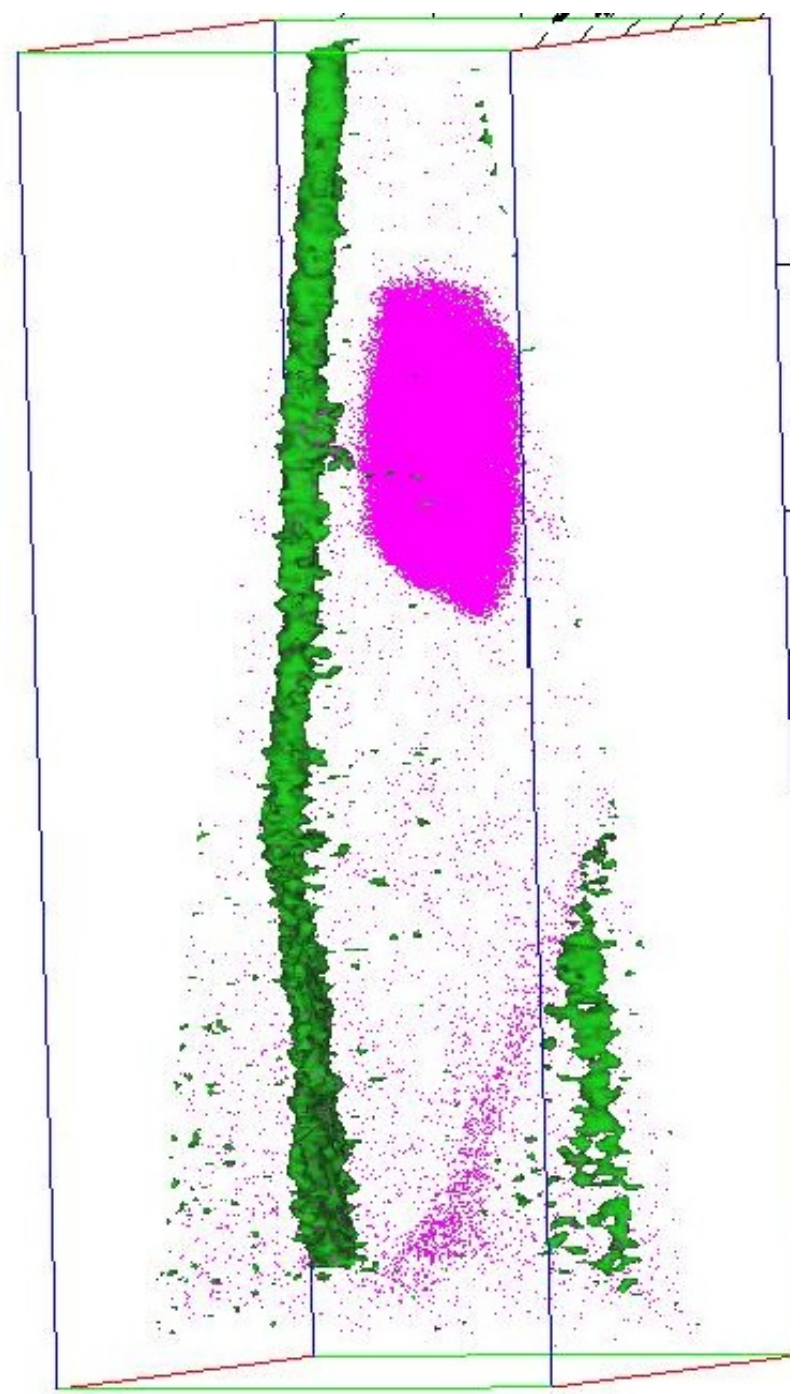

Figure 1: Iso-concentration surface of 3.2 at\% $\mathrm{Sn}$ (green) displayed to show retention to a crystallographic pole in the metal of an LK3 sample. The presence of Fe in an SPP as well as grain boundary segregation of Fe is also rendered (purple). Size of the box is $250 \times 100 \times 100 \mathrm{~nm}^{3}$. 
The marked Sn-rich volume in the analysis contains a local Sn concentration exceeding 3.2 at\%, which is significantly higher than the average concentration. This reflects retention of $\mathrm{Sn}$ at the pole. As the analysis traverses a grain boundary, here made visible by displaying Fe atoms (purple), the pole moves in the analysis as the crystallographic orientation is different in the lower grain. For this reason some care has to be taken when studying the Sn distribution in the metal.

\section{$1 \mu$ m oxide thickness}

A total of 83 samples with $1 \mu \mathrm{m}$ oxide thickness were analyzed, with approximately equal numbers of LK2 and LK3. No significant difference in Sn distribution between LK2 and LK3 could be detected. A typical Sn distribution around the metal-oxide interface in a $1 \mu \mathrm{m}$ oxide sample is presented in Figure 2.

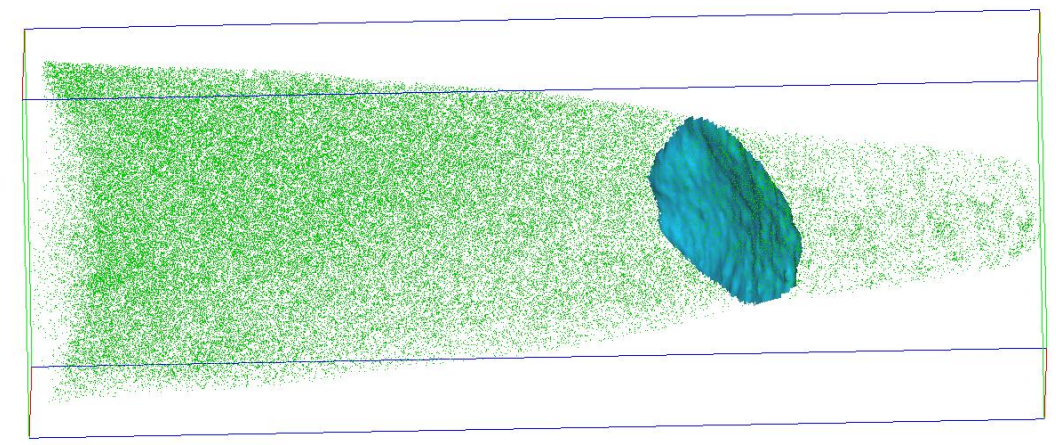

Figure 2: Sn distribution in the metal (left) and in the oxide (right) close to the metal-oxide interface of an LK3 material with $1 \mu \mathrm{m}$ oxide thickness. The blue iso-concentration surface is drawn at an oxygen concentration of 55 at $\%$ and no sub-oxide was present at the interface. Size of the box is $250 \times 90 \times 90 \mathrm{~nm}^{3}$.

No segregation to the interface is seen, confirming the observations made by Wei et al. [3]. It is reasonable to assume that $\mathrm{Sn}$ is incorporated in the oxide in the same state as its metal position, i.e. substitutionally dissolved in the $\mathrm{ZrO}_{2}$ bulk. However, indications of minor clustering of Sn atoms are present in the oxide in the vicinity of the interface in Figure 2.

An atomic map including the oxide a few hundred nanometers away from the interface is presented in Figure 3. 


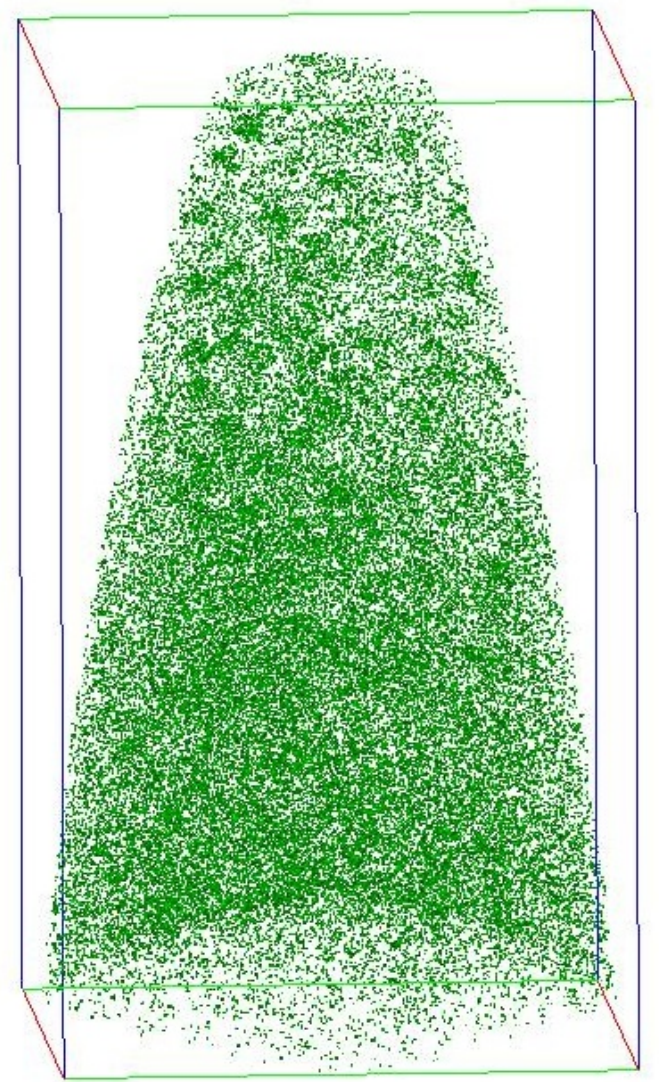

Figure 3: Sn distribution in the oxide some $200 \mathrm{~nm}$ away from the metal oxide interface in an LK3 material with $1 \mu \mathrm{m}$ oxide thickness. Size of the box is $140 \times 80 \times 80 \mathrm{~nm}^{3}$.

In this region of the oxide it is clear that $\mathrm{Sn}$ is no longer distributed homogenously, but local variations are clearly visible. Clusters with a size of approximately $5 \mathrm{~nm}$ are observed. Within these clusters, local Sn concentrations of up to 10 atomic $\%$ are measured.

\section{$2 \mu$ m oxide thickness}

Five APT analyses of the LK3 material and three of the LK2 material with $2 \mu \mathrm{m}$ oxide thicknesses were performed. No significant difference in Sn distribution between the two materials could be found. A typical Sn distribution around the metal-oxide interface is presented in Figure 4. 


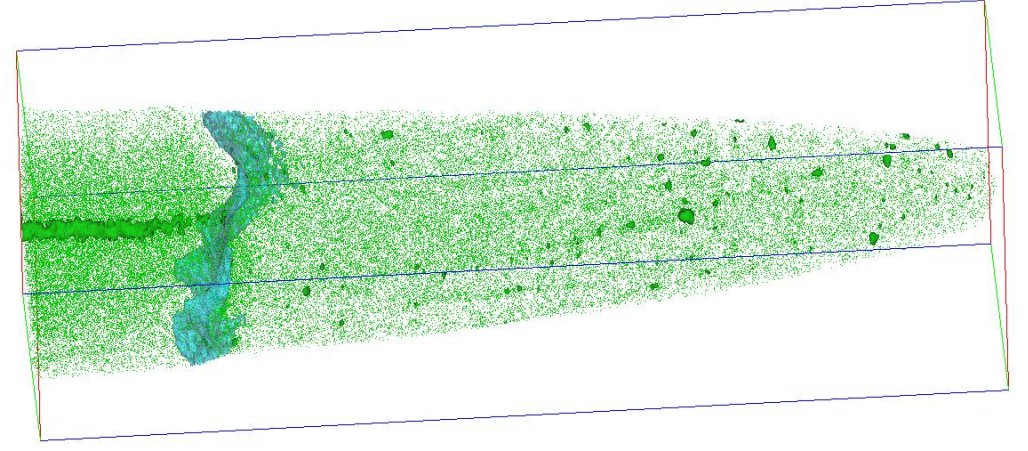

Figure 4: Sn distribution around the metal-oxide interface (blue) of LK2 material with $2 \mu$ m oxide thickness. Green volumes contain more than 4.5 at \% Sn. An iso-concentration surface for 55 at $\%$ oxygen is shown, with oxygen saturated metal to the left and $\mathrm{ZrO}_{2}$ to the right. No sub-oxide phase is present at the interface. Size of the box is $250 \times 100 \times 100 \mathrm{~nm}^{3}$.

The metal-oxide interface is presented as an iso-concentration surface (blue), with the metal to the left and the oxide to the right. A pole is visible in the metal starting at the interface. Green volumes have a local Sn concentration in excess of 4.5 atomic $\%$ and are up to approximately $5 \mathrm{~nm}$ in size. Some of the small Sn particles appear to be aligned in a planar feature in the oxide that may correspond to a grain boundary. The size and number density of these clusters appear to increase with increasing distance from the metal.

\section{$9 \mu$ m oxide thickness}

20 APT samples were analyzed of the LK2 material with $9 \mu$ m oxide thickness. 17 of these analyses were LK2 material and 3 were LK3 material. Again, no significant difference could be seen between the two materials with respect to the Sn distribution. A sub-oxide of composition $\mathrm{ZrO}$ with a thickness of several hundred nanometers is always present at the metal-oxide interface. The Sn distribution in the sub-oxide is shown in Figure 5.

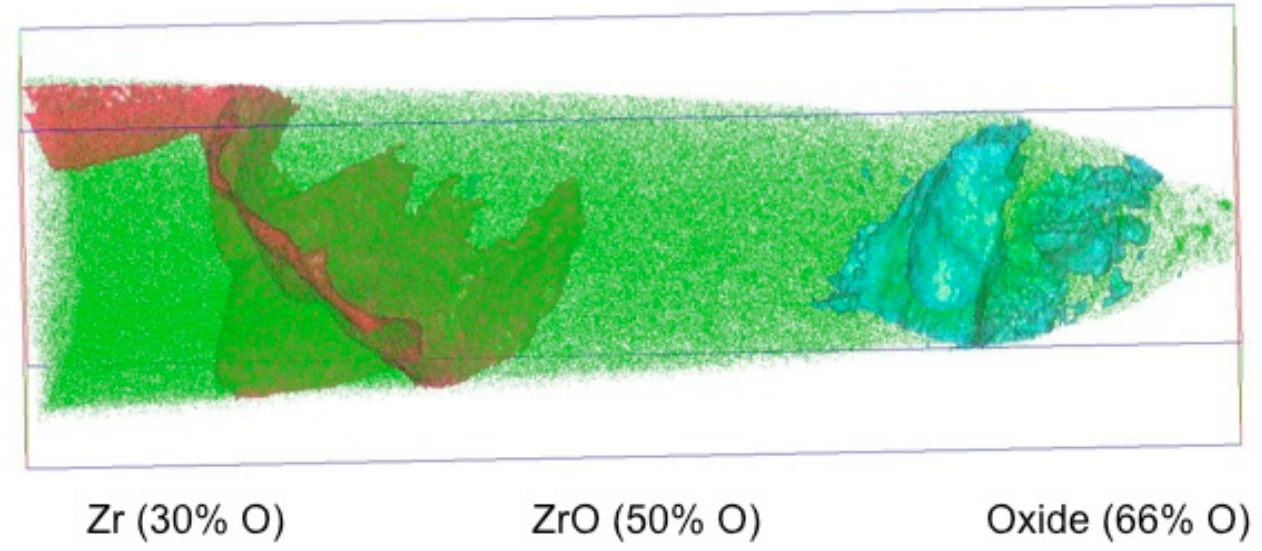

Figure 5: Sn distibution (green) in and around the sub-oxide of the $9 \mu \mathrm{m}$ oxide thickness material (LK2). The metal-suboxide interface is indicated in red and the suboxide-oxide interface is indicated in blue. The size of the box is $445 \times 130 \times 130 \mathrm{~nm}^{3}$. 
No clustering in the sub-oxide was observed, whereas signs of clustering can be seen in the $\mathrm{ZrO}_{2}$. An atomic map of the $\mathrm{Sn}$ distribution in bulk $\mathrm{ZrO}_{2}$ some $300 \mathrm{~nm}$ away from the metal-oxide interface is presented in Figure 6.

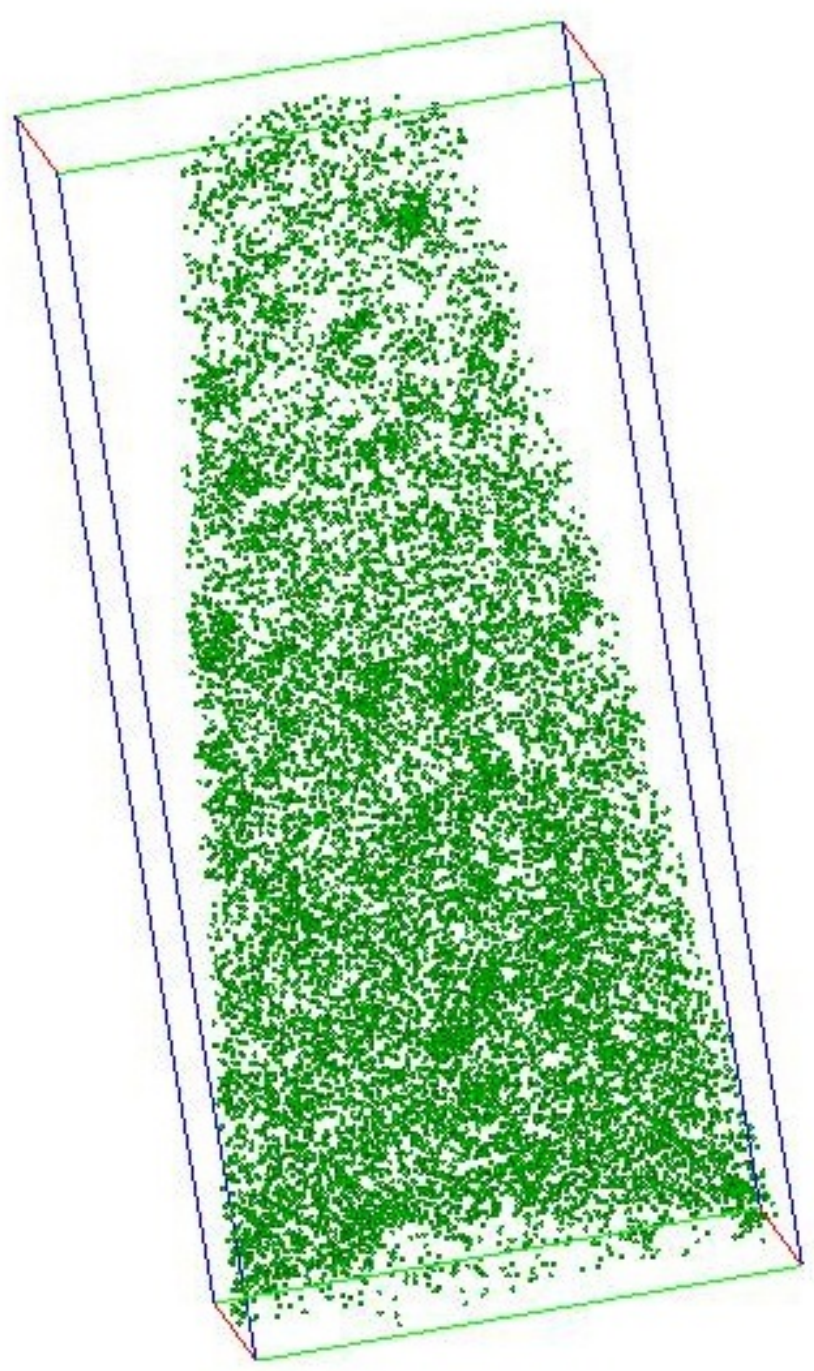

Figure 6: Sn distribution in the oxide some $300 \mathrm{~nm}$ away from the metal-oxide interface of an LK2 material with $9 \mu \mathrm{m}$ oxide thickness. Size of the box is $100 \times 50 \times 50 \mathrm{~nm}^{3}$.

Again, the local concentrations within $5 \mathrm{~nm}$ sized clusters exceed 10 atomic $\%$. A somewhat larger Sn precipitate is presented in Figure 7. 

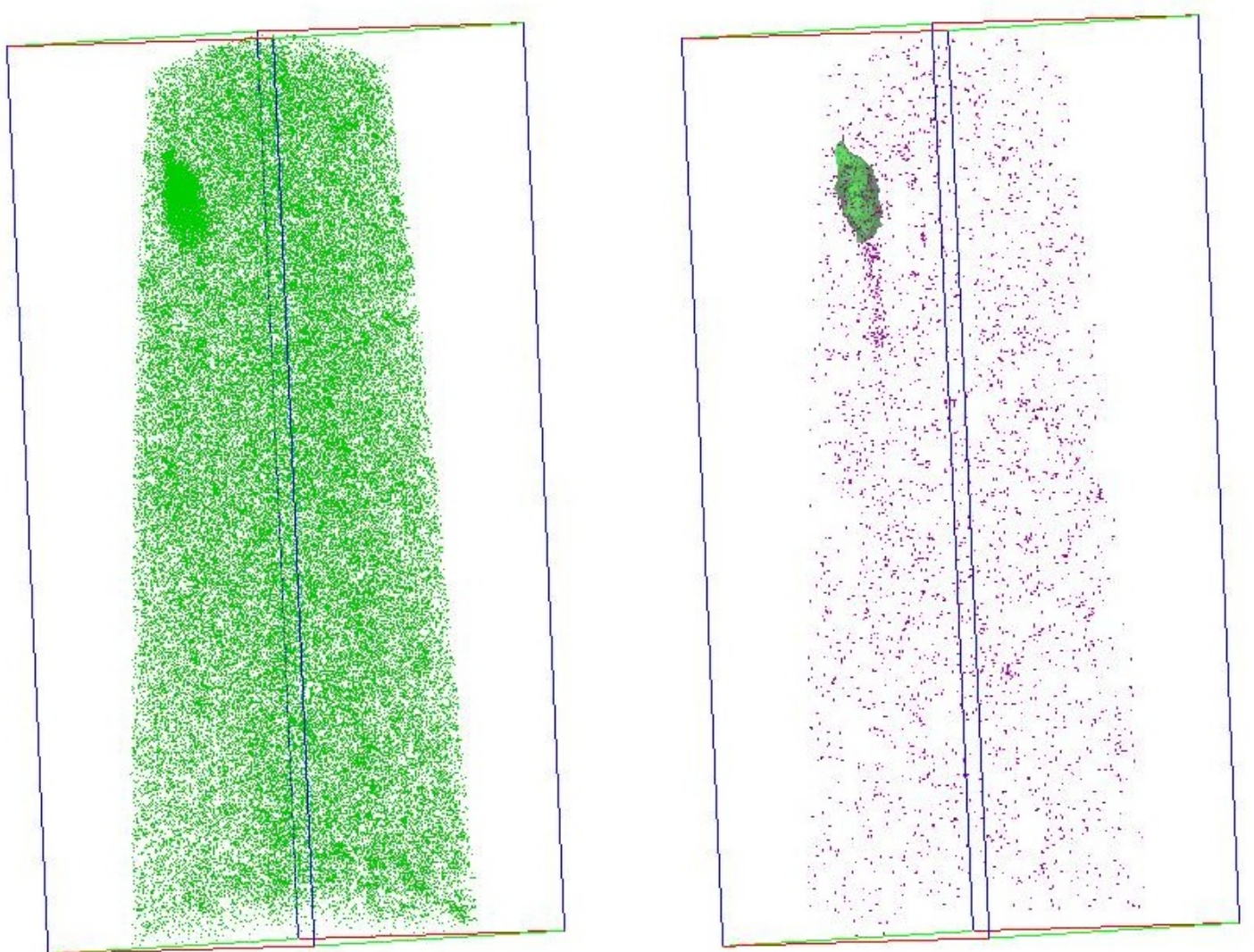

Figure 7: Left: Sn precipitate in the oxide some $400 \mathrm{~nm}$ away from the metal-oxide interface in an LK2 material with $9 \mu$ m oxide thickness. Right: The same analysis with Fe atoms rendered (purple) to show a grain boundary under the particle (green). Size of the boxes is $250 \times 100 \times 100 \mathrm{~nm}^{3}$.

The size of the particle is approximately $20 \mathrm{~nm}$. In Figure 7, to the right, the same reconstruction is displayed rendering the Fe distribution in the vicinity of the precipitate. We have previously shown that Fe can be located in oxide grain boundaries [15], and as Fe is found along a planar feature in direct contact with the precipitate it is reasonable to assume that it has nucleated at the boundary. The particle appears to be somewhat elongated along the direction of the boundary, which could be the result of faster grain boundary diffusion.

A concentration profile (proximity histogram) across the particle-oxide interface is presented in Figure 8. 


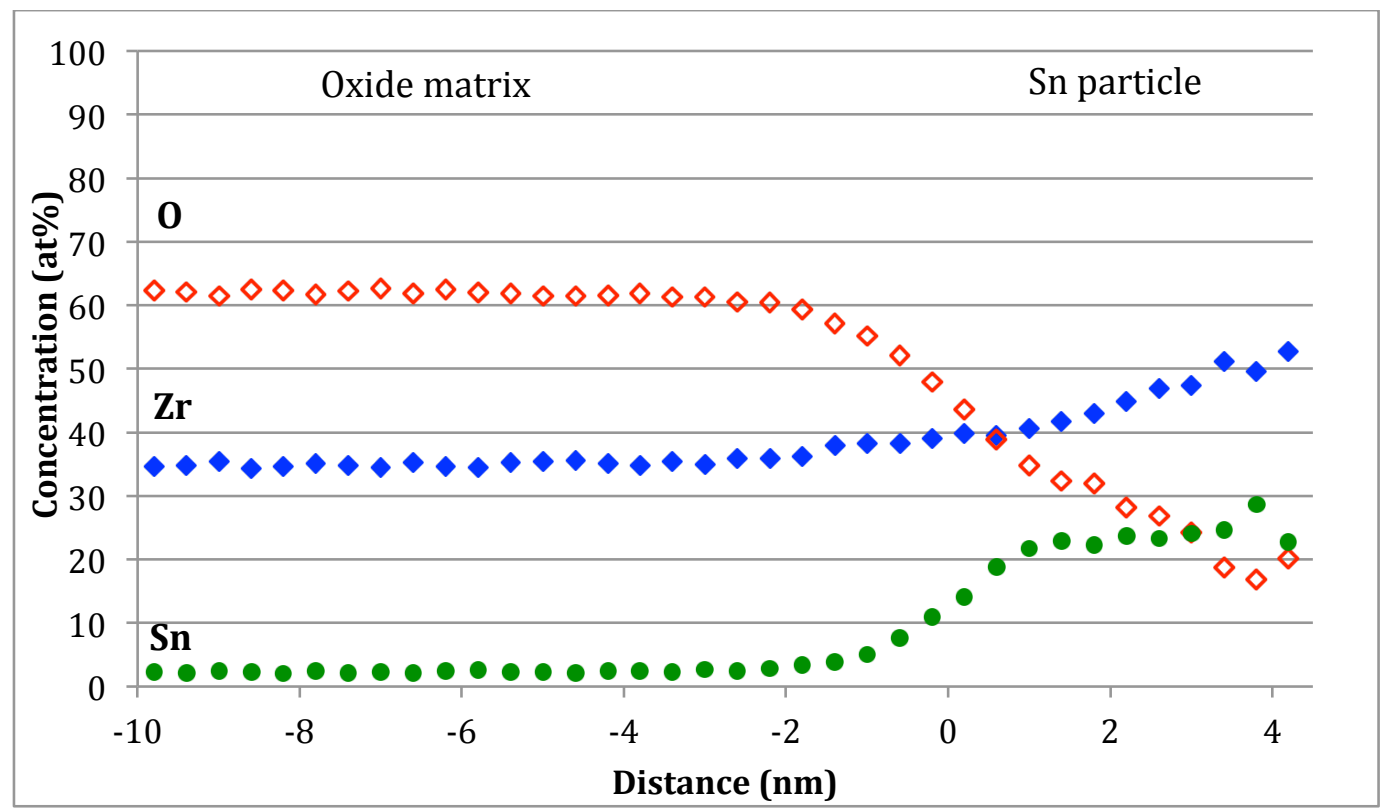

Figure 8: Concentration profile across the boundary between the Sn particle in Figure 7 and the surrounding $\mathrm{ZrO}_{2}$ matrix.

The interior of the precipitate has a maximum Sn concentration of approximately 25 at $\%$. The measured oxygen content in the core of the particle is less that $20 \%$, suggesting that it is metallic, or on the way to become metallic. Another Sn particle, with an even lower oxygen content, was found in the oxide at a similar distance from the metal-oxide interface. The particle and its composition are shown in Figure 9. No such Sn particles have been found in the metal, indicating that they are formed within the oxide.

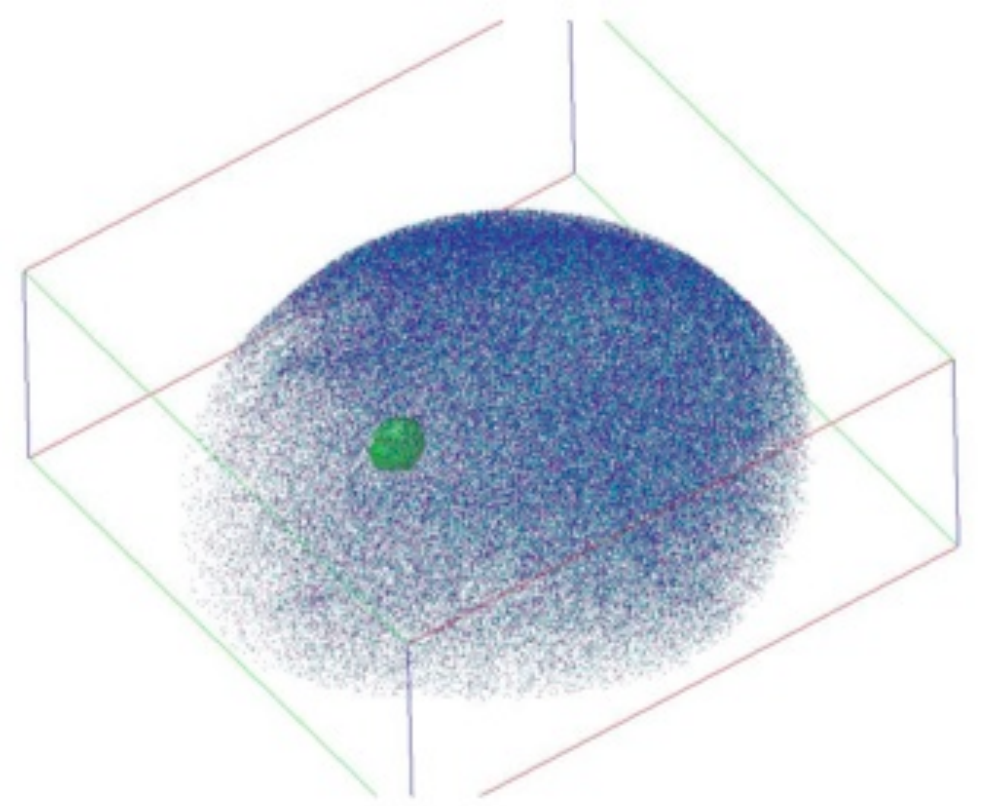

\begin{tabular}{|c|c|}
\hline $\mathrm{Sn}$ & $72.5 \%$ \\
\hline $\mathrm{O}$ & $15.4 \%$ \\
\hline $\mathrm{Zr}$ & $7.6 \%$ \\
\hline $\mathrm{Cr}$ & $2.0 \%$ \\
\hline $\mathrm{Ni}$ & $1.5 \%$ \\
\hline $\mathrm{Fe}$ & $1.0 \%$ \\
\hline
\end{tabular}

Figure 9: Sn particle (green) in the oxide (blue) approximately $400 \mathrm{~nm}$ from the metal oxide interface, and its composition. The size of the box is $115 \times 115 \times 45 \mathrm{~nm}^{3}$. 
Some minor segregation of Sn to a planar feature that is likely a grain boundary was found in one analysis (see Figure 10).

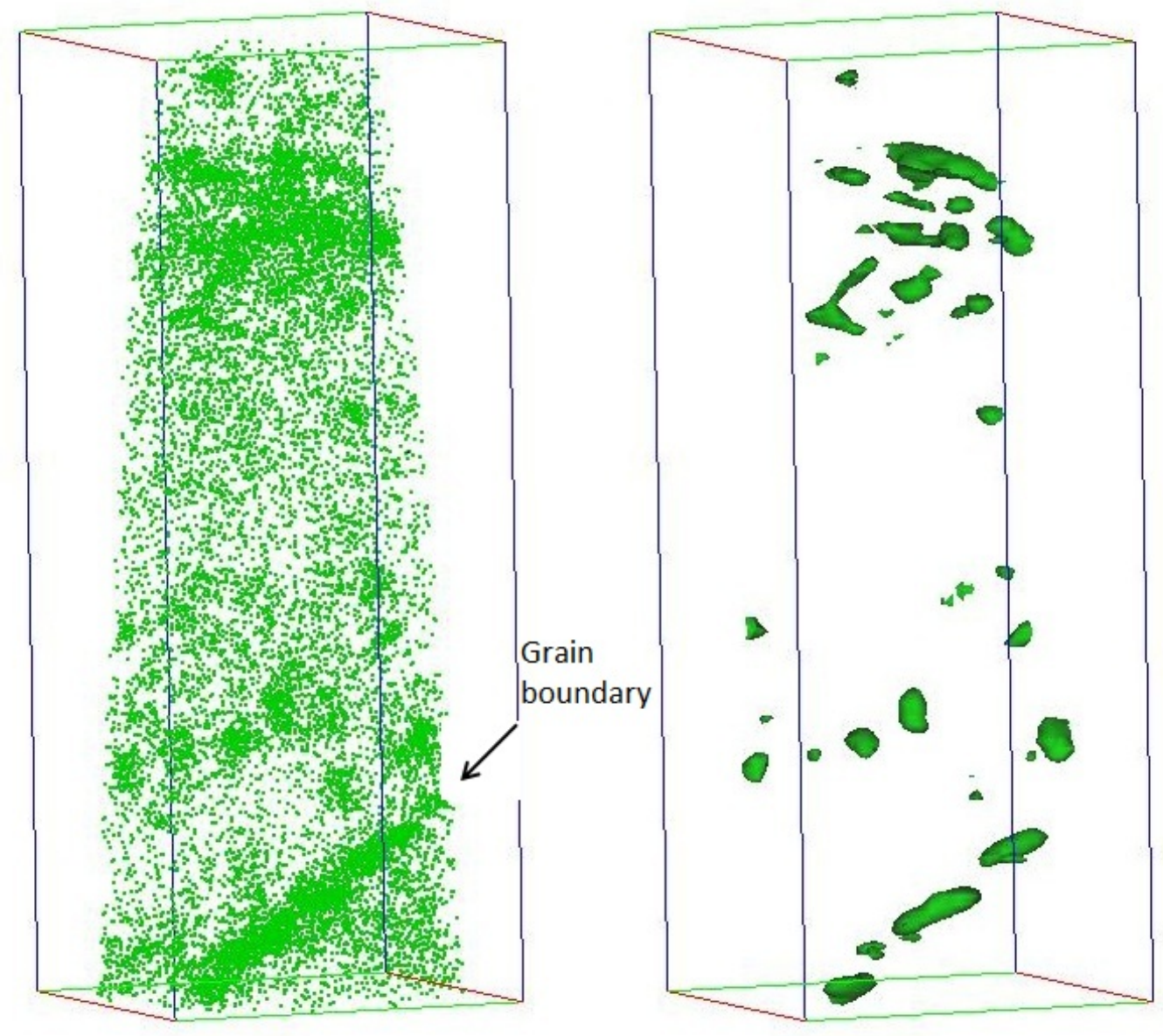

Figure 10: left: Sn clustering in a grain boundary in the oxide of an LK2 sample of $9 \mu$ m oxide thickness, $250 \mathrm{~nm}$ from the metal-oxide interface. To the right: volumes with a local Sn concentration in excess of 5 at\% are rendered. Size of the boxes is $120 \times 50 \times 50 \mathrm{~nm}^{3}$.

Interestingly, $\mathrm{Sn}$ is not distributed homogeneously in the grain boundary plane, but is enriched in clusters along the boundary. None of the analyses of $9 \mu \mathrm{m}$ oxides showed significant rejection of $\mathrm{Sn}$ by the oxide front.

\section{Discussion}

The difference in Sn precipitate (or cluster) size and chemistry when comparing thinner and thicker oxides indicates that the nucleation process is ongoing throughout the corrosion process and equilibrium has likely not been reached $400 \mathrm{~nm}$ away from the metal-oxide interface. Slow $\mathrm{Sn}$ diffusion in the oxide scale is likely to limit the growth of the particles. It is reasonable to assume that the process is significantly faster in nuclear reactors, where diffusion is greatly enhanced by the creation of vacancies from neutron irradiation. We therefore believe that irradiated material must be studied in order to understand the inreactor corrosion process to a satisfactory level. 
The tendency for nucleation and growth of metallic Sn particles in the oxide scale can be understood from an Ellingham diagram [16]. Sn occupies substitutional sites in the $\alpha$-Zr lattice. As no significant rejection by the oxide front is evident, $\mathrm{Sn}$ is also incorporated in the oxide (or sub-oxide) at substitutional sites. This means that like the host lattice $\mathrm{Zr}$ atoms, $\mathrm{Sn}$ is oxidized to a 4+ state immediately upon inclusion in the oxide. At the oxygen activity levels in the vicinity of the metal-oxide interface, metallic $\mathrm{Sn}$ is the most stable species. Thus, a driving force for precipitation exists directly after inclusion in the oxide as Sn strives to remain in a metallic state. Using an Ellingham diagram, one may predict that this precipitation process is ongoing throughout most of the barrier oxide layer (see Figure 11). Metallic $\mathrm{Sn}$ is stable up to $\mathrm{O}_{2}$ partial pressures of up to $10^{-31}$ bar [16], which means that its electrochemical behavior in the oxide should be similar to that of $\mathrm{Ni}$ and Fe. Precipitates in Zircaloy-2 and Ziracloy-4 are known to remain metallic for a reasonably long time in the oxide layer, oxidizing only when oxygen activity is high close to the porous outer oxide $[17,18]$. It is logical to assume that the driving force for precipitation of metallic Sn clusters exists across most of the barrier layer.

Since we believe that the reason for precipitation in the oxide is electrochemical in nature and not related to solubility, Sn will not primarily end up in the $\mathrm{ZrO}_{2}$ grain boundaries. Instead it must nucleate into metallic precipitates in order to achieve a metallic state. This is evident when the Sn distribution a few hundred nanometers away from the metal-oxide interface is analyzed. The nucleation and growth of metallic Sn particles is likely inhibited by slow diffusion in the $\mathrm{ZrO}_{2}$ lattice. Therefore particles can be elongated in the direction of a grain boundary, as is the case in Figures 7 and 10, presumably due to faster diffusion along the boundary. Growth is likely also constrained by diffusion of $\mathrm{Zr}$, and to a lesser extent of $\mathrm{O}$, out of the particles. The oxygen activity is clearly far too high to allow for metallic $\mathrm{Zr}$ is this region. The particle in Figure 7 is therefore probably not at equilibrium, so as to form an intermetallic phase, but is in the process of being depleted in $\mathrm{Zr}$ and $\mathrm{O}$ so that a pure metallic Sn precipitate will be formed. 


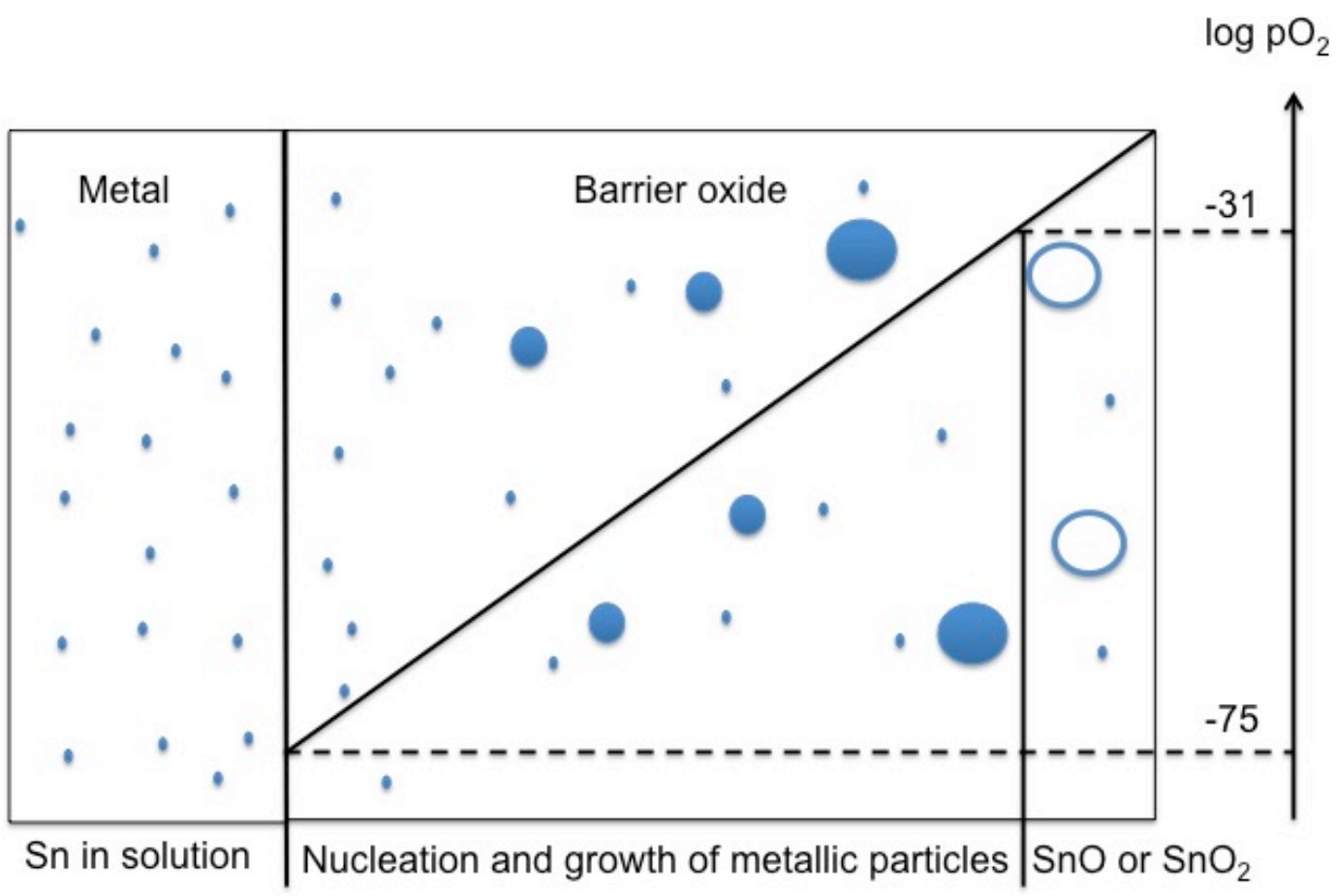

Figure 11: Schematic diagram of the dynamics for $\mathrm{Sn}$ across the barrier layer. $\mathrm{Sn}$ is homogeneously distributed in the $\mathrm{Zr}$ metal matrix. When $\mathrm{Zr}$ starts to oxidize, metallic $\mathrm{Sn}$ is still the stable species, giving a driving force for nucleation and growth that is limited by diffusion. When the oxygen activity is high enough in the outer parts of the barrier layer, $\mathrm{SnO}$ or $\mathrm{SnO}_{2}$ is stable, either dissolved or in small precipitates.

Our interpretation is that the rate of oxidation becomes very important for the kinetics of Sn precipitation. If the oxidation rate is very slow, as it is just before the transition occurs, there will be more time for Sn to move before it enters the outer regions of the barrier layer where the oxygen activity is high enough to allow for $\mathrm{SnO}_{2}$ to be stable substitutionally. Immediately after transition, however, when the oxidation rate is fast, Sn may not have time to precipitate before the oxygen activity is sufficiently high to make the $\mathrm{SnO}_{2}$ stable. Thus, we expect to see more extensive Sn precipitation in $\mathrm{ZrO}_{2}$ formed before transition, than just after. There is no obvious reason for these results not being applicable to other Sn-bearing zirconium alloys such as ZIRLO or Zircaloy-4.

This nucleation and growth process of metallic $\mathrm{Sn}$ particles may also help explain the decrease in tetragonal $\mathrm{ZrO}_{2}$ fraction away from the metal-oxide interface. Sn precipitation will deplete surrounding $\mathrm{ZrO}_{2}$ grains of $\mathrm{Sn}$, thus chemically destabilizing tetragonal $\mathrm{ZrO}_{2}$ gradually as the oxidation proceeds. Godlewski et al. made detailed measurements of tetragonal fraction as a function of oxidation time using Raman spectroscopy, and were able to correlate this with the transition mechanism [19]. These results are in good agreement with our mechanism for Sn depletion of chemically stabilized tetragonal grains being responsible for the phase transformation to monoclinic. This argumentation is strengthened further by the more detailed studies of tetragonal phase fraction carried out by Wei and Frankel $[3,4]$. 


\section{Conclusions}

The evolution of the Sn distribution during corrosion of Ziracloy-2 has been investigated using atom probe tomography. After occupying substitutional solution sites in the $\mathrm{Zr}$ matrix, $\mathrm{Sn}$ is observed to form clusters of a size of a few nanometers upon incorporation in the oxide. These clusters grow to form 20 nanometer sized metallic particles as the oxidation proceeds, inhibited only by slow diffusion in the $\mathrm{ZrO}_{2}$ lattice. We interpret this as being a result of noble $\mathrm{Sn}$ atoms having a driving force to remain metallic at the relatively low oxygen activities close to the metal-oxide interface. At substitutional sites in $\mathrm{ZrO}_{2}$, Sn has to be oxidized to a $4+$ state. Thus, formation of metallic particles devoid of oxygen is stimulated. The process of clustering and particle formation depletes the surrounding $\mathrm{ZrO}_{2}$ grains of $\mathrm{Sn}$, which may lead to transformation of chemically stabilized tetragonal phase to monoclinic $\mathrm{ZrO}_{2}$.

\section{Acknowledgements}

The authors would like to thank Ms. Haiping Lai for assistance with TEM. Westinghouse Electric Sweden AB, Sandvik Materials Technology AB, Vattenfall $\mathrm{AB}$ and the Swedish Research Council are gratefully acknowledged for their financial support.

[1] J. Smith, J. Electrochem. Soc. 112 (1969) 560-567

[2] A.M. Garde, S.P. Pati, M.A. Krammen, G.P. Smith, R.K. Kramer, in: Zirconium in the Nuclear Industry: 10 $0^{\text {th }}$ International Symposium, ASTM STP 1245 (1993) 760-778

[3] J. Wei, P. Frankel, E. Polatidis, M. Blat, A. Ambard, R.J. Comstock, L. Hallstadius, D. Hudson, G.D.W. Smith, C.R.M. Grovenor, M. Klaus, R.A. Cottis, S. Lyon, M. Preuss, Acta Mater. 61 (2013) 4200-4214

[4] P. Frankel, J. Wei, E. Francis, S. Lyon, R.A. Cottis, M. Preuss, N. Ni, S. LozanoPerez, C.R.M. Grovenor, R. J. Comstock, L. Hallstadius, A. Ambard, M. Blat-Yrieix, presentation by P. Frankel at the Zirconium in the Nuclear Industry: $17^{\text {th }}$ International Symposium, Hyderabad, India (2013)

[5] K. Takeda, H. Anada, in: Zirconium in the Nuclear Industry: $12^{\text {th }}$ International Symposium, ASTM STP 1354 (2000) 592-608

[6] D. Pêcheur, V. P. Filippov, A.B. Bateev, J.J. Ivanov, in: Zirconium in the Nuclear Industry: 13 ${ }^{\text {th }}$ International Symposium, ASTM STP 1423 (2002) 135-153

[7] H. Anada, K. Takeda, S, Nasu, Y. Kobayashi, T. Nakamichi, in: Zirconium in the Nuclear Industry: $13^{\text {th }}$ International Symposium, ASTM STP $1423,2002,154-168$

[8] Y. Dong, A.T. Motta, E.A. Marquis, J. Nucl. Mater. 442 (2013) 270-281

[9] T. Andersson, T. Thorvaldsson, A. Wilson, A.M. Wardle, Improvements in

Water Reactor Fuel Technology and Utilization, IAEA, Vienna, Austria, (1987), 435-449

[10] P. Tejland, H.-O. Andrén, G. Sundell, M. Thuvander, B. Josefsson, L. Hallstadius, M. Ivermark, M. Dahlbäck, Zirconium in the Nuclear Industry: 17 th International Symposium, In Press

[11] M.K. Miller, K.F. Russell, G.B. Thompson, Strategies for Fabricating Atom Probe Specimens with a Dual Beam FIB. Ultramicroscopy 102 (2005) 287-298 
[12] K.F. Thompson, D.F. Lawrence, D.J. Larson, J.D. Olson, T.F. Kelly, B. Gorman, Ultramicroscopy 107 (2007) 131-139

[13] B. Wadman, H.-O. Andrén, L.K.L. Falk, Colloque de Physique 50 (1989) 303308

[14] B. Wadman, H.-O. Andrén, U. Rolander, Journal de Physique 49 (1988) 323327

[15] G. Sundell, M. Thuvander, H.-O. Andrén, Corr. Sci. 65 (2012) 10-12

[16] D. Pêcheur, F. Lefebvre, A. Motta, C. Lemaignan, D. Charquet, in: Zirconium in the Nuclear Industry: $10^{\text {th }}$ International Symposium, ASTM STP 1245, 1994, 687708

[17] F. Garzarolli, H. Seidel, R. Tricot, J.P. Gros, in: Zirconium in the Nuclear Industry: $9^{\text {th }}$ International Symposium, ASTM STP 1991, 395-415

[18] D. Pêcheur, F. Lefebvre, A.T. Motta, C. Lemaignan, J. F. Wadier, J. Nucl. Mater. 189 (1992) 318-332

[19] J. Godlewski, J.P. Gros, M. Lambertin, J.F. Wadier, H. Weidinger, in: Zirconium in the Nuclear Industry: 9 ${ }^{\text {th }}$ International Symposium, ASTM STP 1132 (1991) 416-436 